LAW RENCE LIVERMORE N A T IO N A L LABORATORY

Neutron Production by Muon Spallation I: Theory

T. Luu, C. Hagmann

November 22, 2006 
This document was prepared as an account of work sponsored by an agency of the United States Government. Neither the United States Government nor the University of California nor any of their employees, makes any warranty, express or implied, or assumes any legal liability or responsibility for the accuracy, completeness, or usefulness of any information, apparatus, product, or process disclosed, or represents that its use would not infringe privately owned rights. Reference herein to any specific commercial product, process, or service by trade name, trademark, manufacturer, or otherwise, does not necessarily constitute or imply its endorsement, recommendation, or favoring by the United States Government or the University of California. The views and opinions of authors expressed herein do not necessarily state or reflect those of the United States Government or the University of California, and shall not be used for advertising or product endorsement purposes.

This work was performed under the auspices of the U.S. Department of Energy by University of California, Lawrence Livermore National Laboratory under Contract W-7405-Eng-48. 


\title{
Neutron Production by Muon Spallation I: Theory
}

\author{
Thomas $\mathrm{Luu}^{1, *}$ and Christian Hagmann ${ }^{1, \dagger}$ \\ ${ }^{1} \mathrm{~N}$-Division, Lawrence Livermore National Laboratory
}

(Dated: November 13, 2006)

We describe the physics and codes developed in the Muon Physics Package. This package is a self-contained Fortran90 module that is intended to be used with the Monte Carlo package MCNPX. We calculate simulated energy spectra, multiplicities, and angular distributions of direct neutrons and pions from muon spallation.

${ }^{*}$ E-mail: tluu@llnl.gov

${ }^{\dagger}$ E-mail: hagmann1@llnl.gov 


\section{INTRODUCTION}

Neutron production via muon spallation is an important process in understanding the role of terrestrial nuclear processes due to cosmic ray interactions. It is a main background contribution to low-energy underground experiments, such as searches for proton decay (Kamiokande) or neutrino oscillations (SNO). At sea level, on the other hand, neutron production is primarily due to neutron and proton spallation. With increasing depth this source quickly loses its dominance as its yield is reduced by 3 orders of magnitude at 10 m.w.e. At this depth most of the neutrons are produced by negative muon capture with a multiplicity of a few per captured neutron. Larger, albeit rarer, neutron bursts are produced by inelastic muon scattering where the available energy can be large. Assessing the frequency and size distribution of the bursts is the topic of this study.

The neutron yields from cosmic muon spallation have been measured by several experiments, though with contradictory results [1-4]. Theoretical calculations $[4,5]$ are hampered by the fact that secondary neutrons can be produced by hadronic interactions with primary neutrons (and primary pions). These processes are hard to quantify analytically. Thus a Monte Carlo treatment is needed to simulate the neutron yields, tracking both the primary neutrons and secondary neutrons that are produced from interactions with primary neutrons and pions. Wang et al. [6] have used the Monte Carlo package FLUKA to this cause, obtaining good agreement with most experiments. However, the source codes for both FLUKA and its muon physics package are not readily available. To understand the relative contributions of various muon physical processes that produce terrestrial neutrons, it is essential to have access to the source codes.

In support of the "Assessment of Long-Dwell, In-Transit Detection System for Cargo Ships", Task 6, we have developed an open source, stand-alone, muon physics package coded as a Fortran90/95 module. It is intended to be used by any Monte Carlo package that is coded in Fortran, such as MCNPX. This Technical Report addresses the details of this physics package. The next section describes the formal theory behind muon spallation and its approximations. In Sect. III we describe our coding logic and list the relevant routines from the physics package. Section IV gives results of our calculations for primary processes. We conclude in Sect. V. A forthcoming companion technical report will give results of simulations that couple this muon physics package with MCNPX. These results will include 


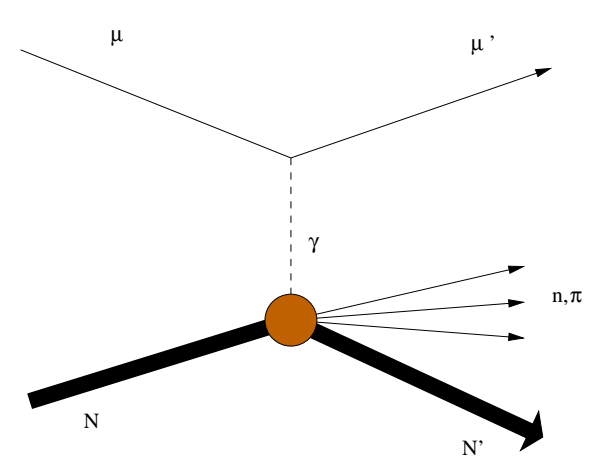

(a)

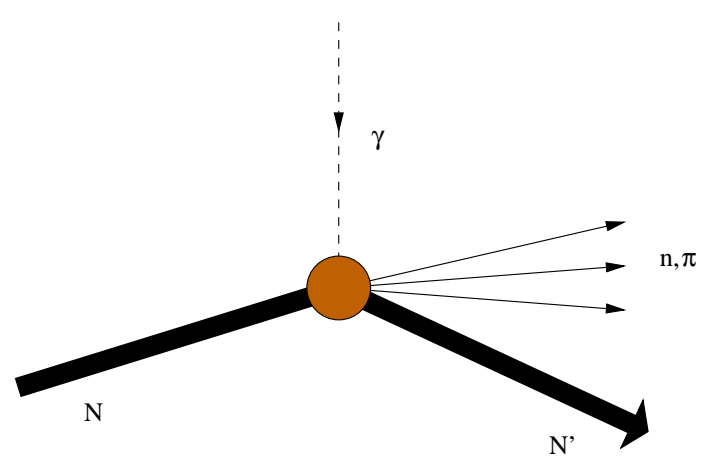

(b)

FIG. 1: Feynman diagrams depicting muon spallation (a) and photo-absoprtion (b). In panel (a) an incoming muon exchanges a virtual photon with a nucleus. Panel (b) shows a real photon being absorbed by the nucleus. The nucleus subsequently emits neutrons or pions.

the effects of secondary processes, such as neutrons produced via primary pions or primary neutrons.

\section{MUON SPALLATION THEORY}

In muon spallation, an incoming muon interacts with a nucleus via an exchange of a virtual photon. The nucleus subsequently emits neutrons (or pions). Figure 1(a) shows a Feynman diagram depicting this scenario. The differential cross section for the inclusive reaction of a charged lepton (see, for example, Ref. [7]), such as the muon, is

$$
\frac{d \sigma}{d E_{f} d \Omega_{f}}=\frac{\alpha^{2}}{q^{4}} \frac{p_{f}}{p_{i}} L^{\mu \nu} W_{\mu \nu}
$$

where $\alpha$ is the fine structure constant, $p_{i}$ and $p_{f}$ are the initial and final lepton momenta, respectively, $q$ is the four-momentum transfer, $L^{\mu \nu}$ is the lepton tensor, and $W^{\mu \nu}$ is the hardronic structure tensor. The lepton tensor is known analytically,

$$
L^{\mu \nu}=2\left[p_{i}^{\mu} p_{f}^{\nu}+p_{i}^{\nu} p_{f}^{\mu}+g^{\mu \nu}\left(m^{2}-p_{i} \cdot p_{f}\right)\right]
$$

where $m$ is the lepton mass. The hadronic tensor, on the other hand, depends on nuclear details that are not know analytically and must be empirically parametrized. It does satisfy certain symmetries, however, which constrains the parametrization. For example, it must 
satisfy the Ward identity $q^{\mu} W_{\mu \nu}=0$, which forces $W_{\mu \nu}$ to take the form

$$
W^{\mu \nu}=W_{1}\left(-g^{\mu \nu}+\frac{q^{\mu} q^{\nu}}{q^{2}}\right)+\frac{W_{2}}{M^{2}}\left(p^{\mu}-\frac{q \cdot p}{q^{2}} q^{\mu}\right)\left(p^{\nu}-\frac{q \cdot p}{q^{2}} q^{\nu}\right),
$$

where $p$ is the incoming four-momentum of the hadron, $M$ its mass, and $W_{1}$ and $W_{2}$ are related to the transverse and longitudinal response functions, respectively. Because of Lorentz

invariance, the response functions can only depend on $q^{2}$ and $q \cdot p$, which are independent variables. With this parametrization, Eq. 1 becomes

$$
\frac{d \sigma}{d E_{f} d \Omega_{f}}=\frac{2 \alpha^{2}}{q^{4}} \frac{p_{f}}{p_{i}}\left(W_{1}\left(q^{2}, q \cdot p\right)\left(q^{2}-2 m^{2}\right)+W_{2}\left(q^{2}, q \cdot p\right)\left(2 E_{i} E_{f}+\frac{q^{2}}{2}\right)\right) .
$$

Though there are various nuclear models that predict the form of the structure functions $W_{i}\left(q^{2}, q \cdot p\right)$, a definitive analytic solution is not known. For extremely relativistic leptons, it can argued that the longitudinal response function is much smaller than the transverse, $W_{2} \ll W_{1}$, and that $q \cdot p \gg q^{2}$. This is the basis of the Weizsäcker-Williams approximation [8, 9], to be described in Sect. II A. This approximation gives a model-independent description of the reaction at high lepton (muon) energies.

A necessary ingredient to formulating the Weizsäcker-Williams approximation is to understand the process shown in Fig. 1(b), where a real photon undergoes photo-absorption and neutrons or pions are subsequently emitted. The total cross section for this reaction is

$$
\sigma_{\gamma}\left(E_{\gamma}\right)=\frac{4 \pi \alpha}{E_{\gamma}} W_{1}\left(q^{2}=0, q \cdot p=E_{\gamma} M\right)
$$

where $E_{\gamma}$ is the photon energy and $W_{1}$ is the same transverse response function used in Eqs. 3 and 4. See, for example, Ref [9] for a thorough derivation of this cross section. Note that since this process involves a real photon, the ensuing cross section resides on the $q^{2}=0$ plane of the transverse response function.

\section{A. Weizsäcker-Williams Approximation}

For highly relativistic charged particles, it can be shown that the dominant part of the inelastic cross section grows as $1 / q^{2}$ for small $q^{2}$ momentum transfers [9]. From symmetry arguments, one can establish the following relations as $q^{2} \rightarrow 0$

$$
\begin{array}{ll}
W_{2}\left(q^{2}, q \cdot p\right)=O\left(q^{2}\right), & \text { for } q^{2} \rightarrow 0 \\
W_{1}\left(q^{2}, q \cdot p\right)=\frac{(q \cdot p)^{2}}{M^{2} q^{2}} W_{2}\left(q^{2}, q \cdot p\right) &
\end{array}
$$


Thus for small momentum transfers, $W_{1} \gg W_{2}$. Classically, this can be viewed as the Lorentz contraction of the electric fields along the transverse direction for accelerating charged particles. The Weizsäcker-Williams approximation (also known as the method of virtual quanta) exploits the similarity between the fields of a rapidly moving charged particle and the fields of a pulse of radiation. This similarity allows one to relate the transverse response function to the total photo-absorption cross section at $q^{2}=0$,

$$
W_{1}\left(q^{2}=0, q \cdot p\right)=\frac{\sqrt{(q \cdot p)^{2}}}{(2 \pi)^{2} \alpha M} \sigma_{\gamma}\left(\frac{q \cdot p}{M}\right),
$$

which is equivalent to Eq. 5 using the relation $q \cdot p=E_{\gamma} M$. Assuming $m \ll E_{i}$ and taking the leading order in $q^{2}$, Eq. 4 becomes

$$
\frac{d \sigma}{d E_{f} d \Omega_{f}}=\frac{\alpha}{q^{2}} \frac{p_{f}}{p_{i}} \frac{E_{\gamma}}{2 \pi^{2}} \sigma_{\gamma}\left(E_{\gamma}\right) .
$$

Following Delorme et al.[5], the solid-angle integral over $\Omega_{f}$ can be performed to give

$$
\frac{d \sigma}{d E_{f}}=\frac{N\left(E_{\gamma}\right) \sigma_{\gamma}\left(E_{\gamma}\right)}{E_{\gamma}}
$$

where

$$
N\left(E_{\gamma}\right)=\frac{\alpha}{\pi}\left[\frac{E_{i}^{2}+E_{f}^{2}}{p_{i}^{2}} \ln \left(\frac{E_{f} E_{i}+p_{i} p_{f}-m^{2}}{m E_{\gamma}}\right)-\frac{\left(E_{i}+E_{f}\right)^{2}}{2 p_{i}^{2}} \ln \left(\frac{\left(p_{i}+p_{f}\right)^{2}}{\left(E_{i}+E_{f}\right) E_{\gamma}}\right)-\frac{p_{f}}{p_{i}}\right] .
$$

Equation 9 can be interpreted as a spectrum of equivalent real photons. Thus the WeizäckerWilliams approximation relates the differential muon cross section to a spectrum of equivalent real photons and the total photo-absorption cross section. The latter term is generally more accessible experimentally than the total muon cross section. Figure 2 shows an example of the equivalent photon spectrum for a certain set of input muon parameters.

In general, the Weizsäcker-Williams approximation only works in regimes where the "offshell" behavior of the exchanged photon is small. This requires that the exchanged photon's energy be above the $\Delta$-resonance of the nucleon, which is approximately $300 \mathrm{MeV}$. Below this threshold, it is expected that neutron production due to muon spallation is small compared to neutron production due to muon bremsstrahlung $[5,6]$.

\section{B. Photo-Absorption Model}

Though experimental data of photo-absorption cross sections are readily available for various nuclei, we have adopted the generalized vector dominance (GVD) model as described 


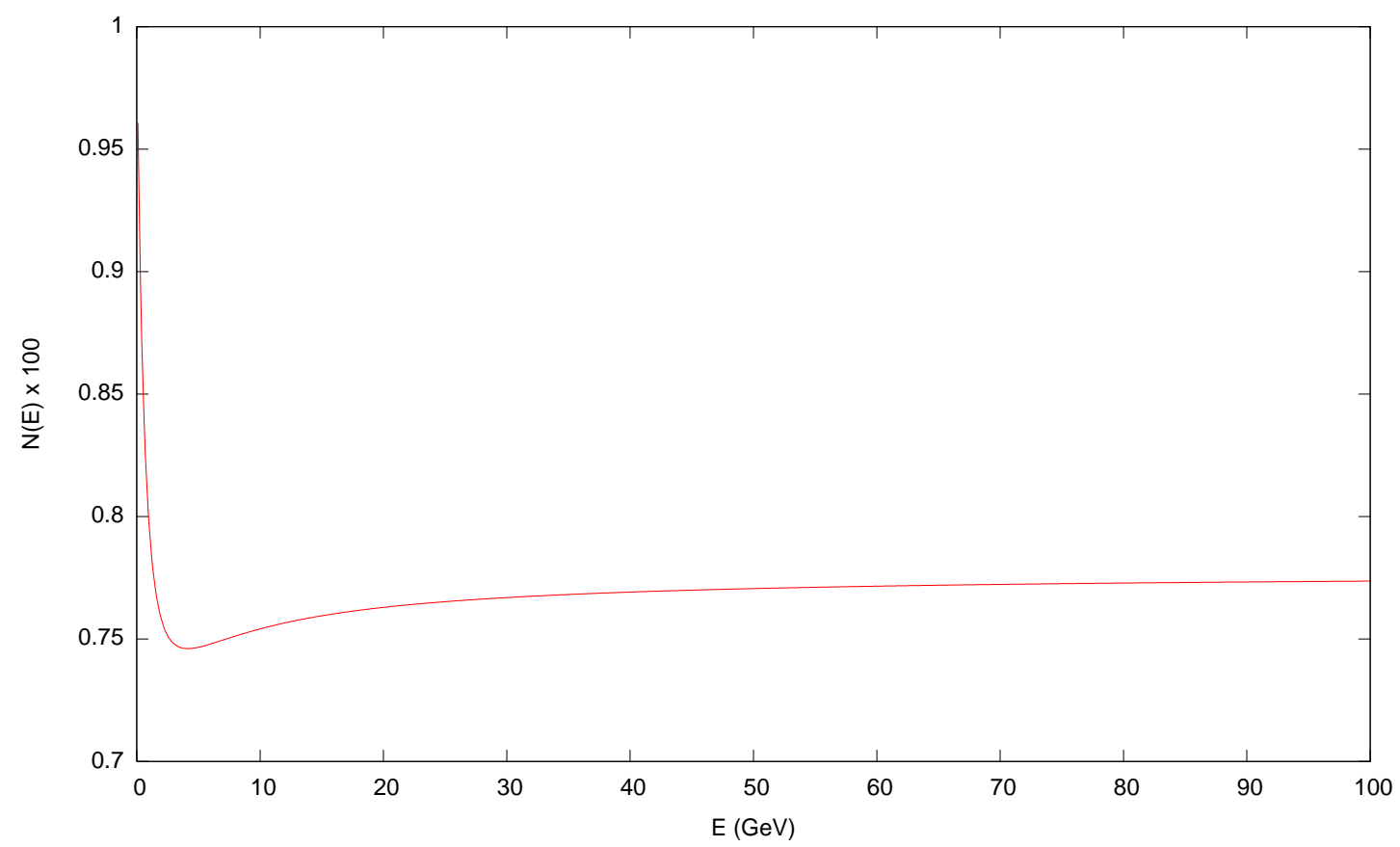

FIG. 2: Spectrum of real photons for final muon relativistic energy $E_{f}=\sqrt{m^{2}+p_{f}^{2}}=2 \mathrm{GeV}$. The energy of the exhanged photon is $E$ (the x axis), and the initial muon relativistic energy $E_{i}=\sqrt{m^{2}+p_{i}^{2}}$ is changed accordingly.

in, for example, Bezrukov and Bugaev[10] to estimate these cross sections. This model gives the total photo-absorption cross section on any nucleus via a closed formula. Thus we avoid the need for storage of experimental data and interpolation routines on the data.

A formal description of the GVD model is beyond the scope of this report, but we list some of its important features: a) it incorporates nucleon shadowing (a well established physical effect), where the total photo-absorption cross section $\sigma_{\gamma A}$ is smaller than $A \sigma_{\gamma n}$, where $\sigma_{\gamma n}$ refers to the total photo cross section on an individual nucleon; b) it uses an optical model of the nucleus that incorporates an off-diagonal GVD model with $\rho$-meson finite-width corrections; and c) it takes into account the growth of hadron cross sections with energy. The cross section in $\mu \mathrm{b}$ on nucleus of atomic number $A$ with photon energy $E$ in $\mathrm{GeV}$ is given by

$$
\sigma_{\gamma A}(E)=A \sigma_{\gamma n}(E)(.75 G(x)+.25)
$$




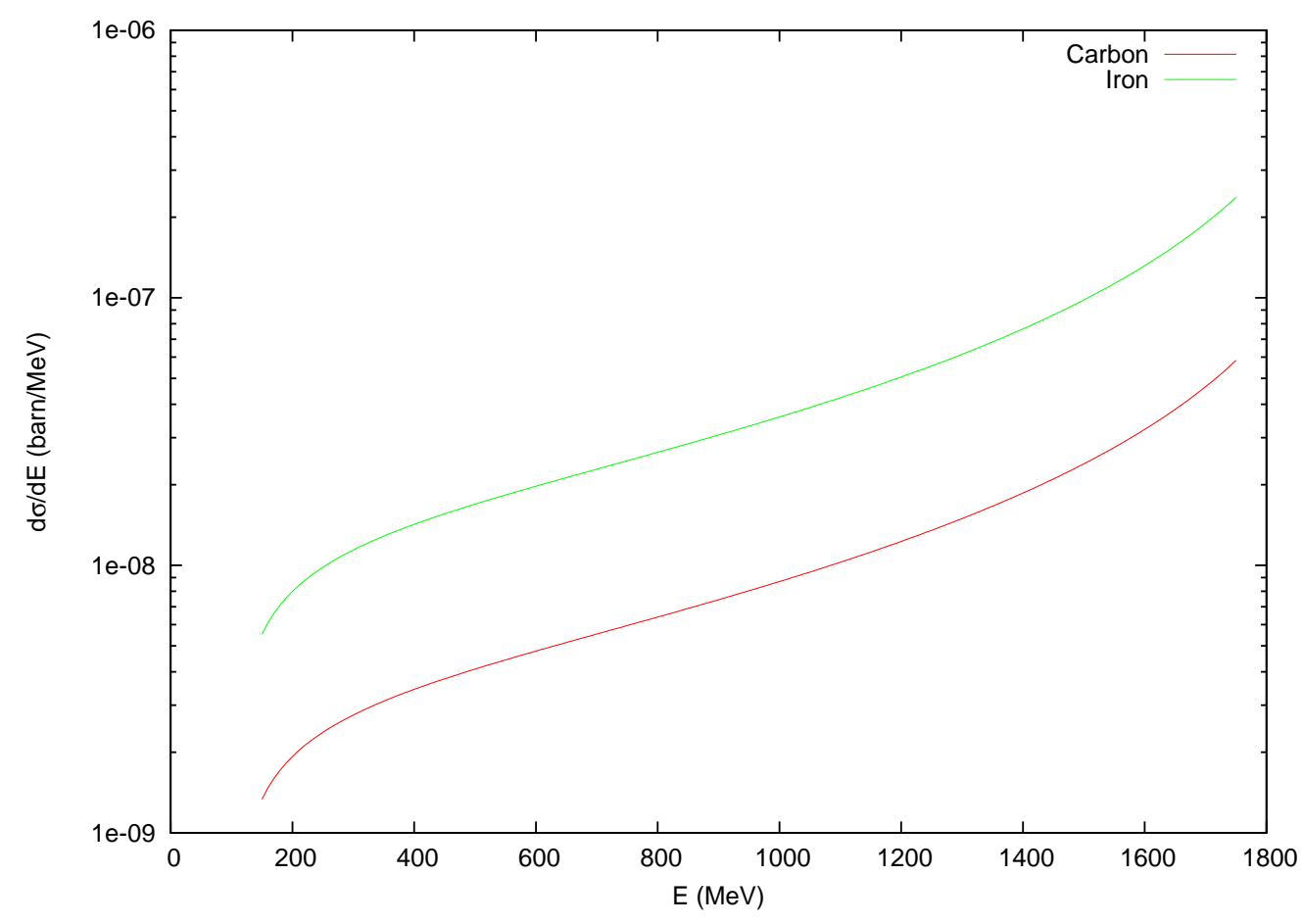

FIG. 3: Differential cross section as a function of outgoing muon relativistic energy $E$ (Eq. 8) for Carbon and Iron. In both cases, the incoming muon relativistic energy was held at $2 \mathrm{GeV}$.

where

$$
\begin{aligned}
x & =.00282 A^{1 / 3} \sigma_{\gamma n}(E) \\
G(x) & =\frac{3}{x^{3}}\left(\frac{x^{2}}{2}-1+e^{-x(1+x)}\right),
\end{aligned}
$$

and the photo-nucleon cross section, $\sigma_{\gamma n}$, is approximated by (in $\mu \mathrm{b}$ )

$$
\sigma_{\gamma n}(E)=114.3+1.647 \ln ^{2}(0.0213 E)
$$

Equation 10 used with Eq. 8 gives the differential cross section of the muon with respect to the outgoing muon energy. Figure 3 shows this quantity for Carbon and Iron. This function is sampled stochastically to obtain the outgoing muon energy, and thus the photon energy transfer to the nucleus.

The total muon cross section is obtained by integrating Eq. 8 over outgoing muon energies, 


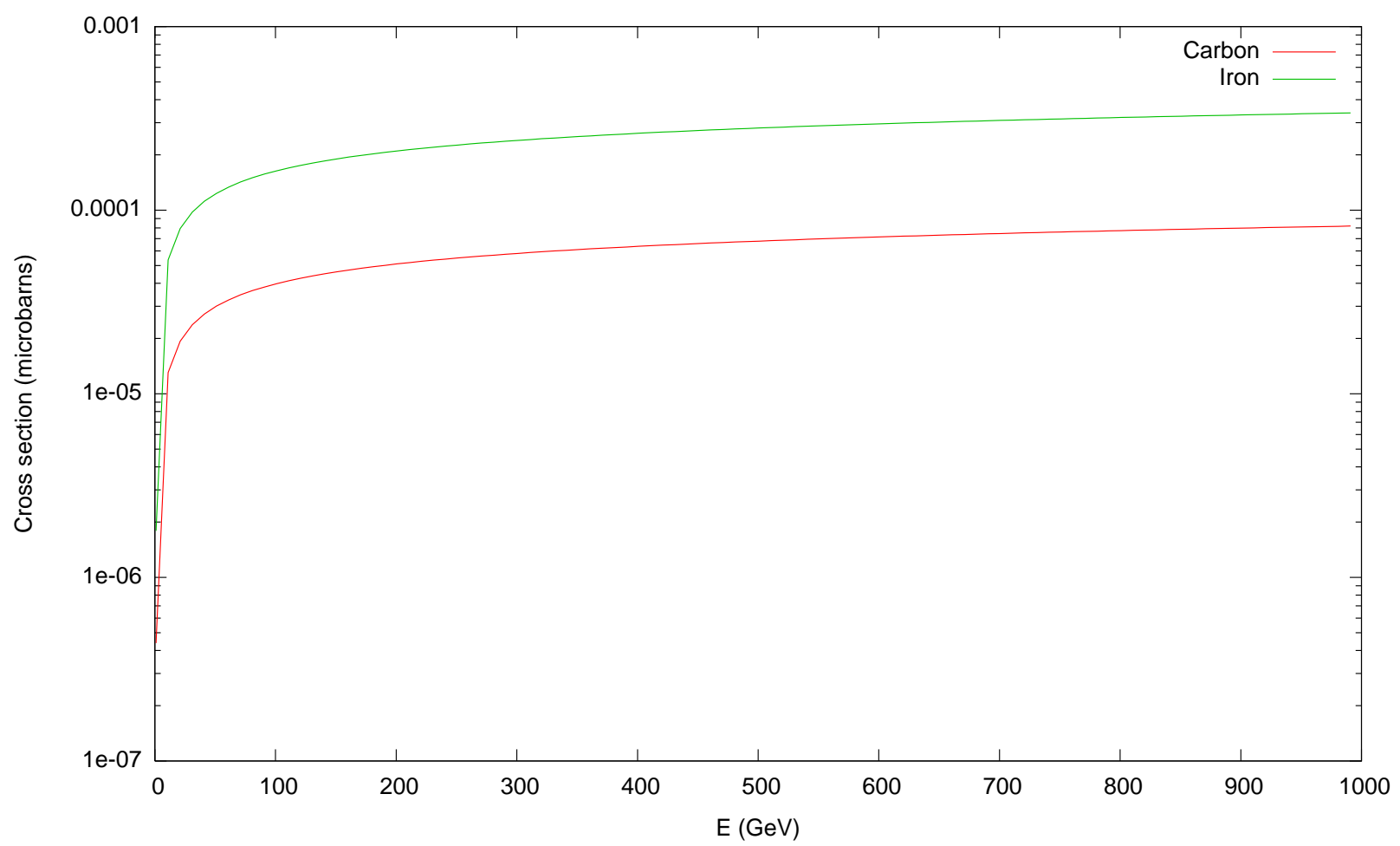

FIG. 4: Total muon cross section (in $\mu \mathrm{b}$ ) for Carbon and Iron as calculated by the WeizsäckerWilliams approximation as a function of incoming muon kinetic energy.

or equivalently, over appropriate exchanged photon energies,

$$
\sigma\left(E_{i}\right)=\int_{E_{\Delta}}^{E_{i}-m} d E_{\gamma} \frac{N\left(E_{\gamma}\right) \sigma_{\gamma}\left(E_{\gamma}\right)}{E_{\gamma}},
$$

where $E_{i}$ is the incoming muon relativisitc energy, $E_{\delta}$ is the delta-resonance energy, and $m$ is the muon mass. Figure 4 shows the total cross section for Carbon and Iron for a range of incoming muon kinetic energies.

\section{Neutron and Pion Multiplicities}

The average particle multiplicity $m$, as obtained via the Weizsäcker-Williams approximation, is given by the expression

$$
m\left(E_{i}\right)=\frac{\int_{E_{\Delta}}^{E_{i}-m_{\mu}} d E_{\gamma} \frac{N\left(E_{\gamma}\right)\left[\bar{m}\left(E_{\gamma}\right) \sigma_{\gamma}\left(E_{\gamma}\right)\right]}{E_{\gamma}}}{\int_{E_{\Delta}}^{E_{i}-m_{\mu}} d E_{\gamma} \frac{N\left(E_{\gamma}\right) \sigma_{\gamma}\left(E_{\gamma}\right)}{E_{\gamma}}}
$$

where $\bar{m}\left(E_{\gamma}\right)$ is the multiplicity of the same particle due to photo-production, $E_{i}$ is the relativistic incoming muon energy, $m_{\mu}$ is the rest mass of the muon, and $E_{\Delta}$ is the energy 


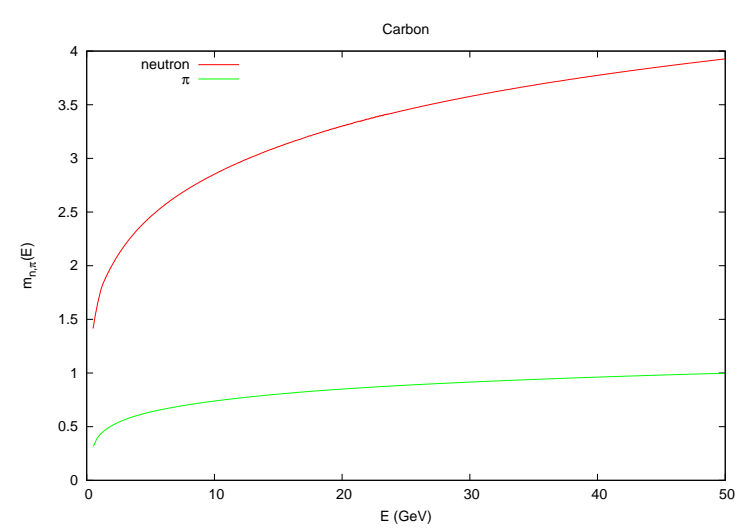

(a)

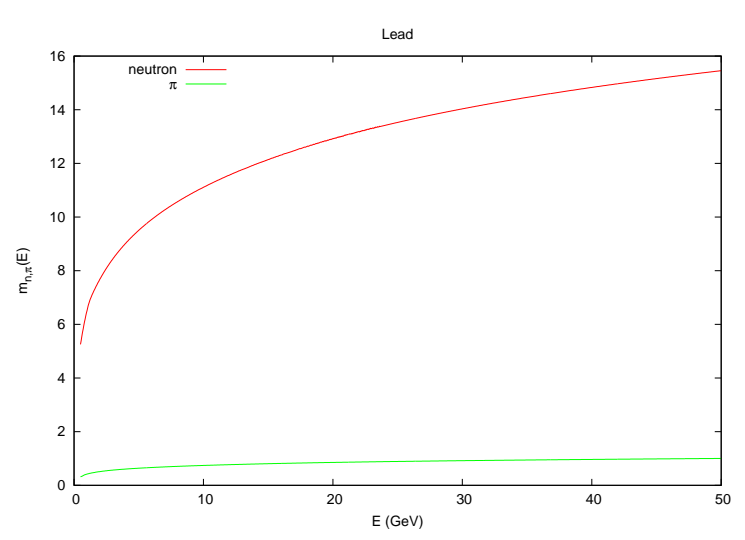

(b)

FIG. 5: Neutron and pion average multiplicities for Carbon (a) and Lead (b).

of the $\Delta$ resonance.

For pion multiplicities, we follow Delorme et al. [5] in constructing $\left[\bar{m}\left(E_{\gamma}\right) \sigma_{\gamma}\left(E_{\gamma}\right)\right]$. This quantity is obtained empirically by first constructing the branching ratio $\operatorname{br}\left(E_{\gamma}\right)$ for photo pion-production from a single nucleon (i.e proton) from existing experimental data [11]. Here it is assumed that the branching ratio for photo production of $\pi^{+}$is the same as $\pi^{-}$ due to the approximate isospin invariance at large photon energies. The multiplicty for photo pion-production off of a nucleus is obtained by multiplying the nucleon $\operatorname{br}\left(E_{\gamma}\right)$ by the photo-absorption cross section for the entire nucleus, as given by Eq. 10 .

We follow Allkofer and Andresen[4] to obtain neutron multiplicities. Here direct experimental data of the quantity $\left[\bar{m}\left(E_{\gamma}\right) \sigma_{\gamma}\left(E_{\gamma}\right)\right]$ is used[12], as well as a modification of the virtual quanta spectrum $N\left(E_{\gamma}\right)$ given by Kessler[13]. The data is available for a select set of nuclei. For nuclei in which there is no experimental data, the quantity $\left[\bar{m}\left(E_{\gamma}\right) \sigma_{\gamma}\left(E_{\gamma}\right)\right]$ is interpolated from existing data. At high energies it is assumed that the value of $\bar{m}(E)$ scales as $E^{1 / 4}$, which seems to give good agreement with existing experimental neutron multiplicities due to muon spallation on Lead [4].

In Fig. 5 we show the average neutron and pion multiplicities on Carbon and Iron. Note the relative insensitivity of the pion multiplicity in the two cases. 


\section{CODE LOGIC AND IMPLEMENTATION}

The muon physics package is coded as a Fortran90 module. It is self-contained. The minimal routines needed to access this package are

double function sigmaMuA_kin(A,Ein)

subroutine scatter_muon(A,Z,Ein,Eout,m,particle_flag)

subroutine get_energies_angle_neutron(n,energy,cos_theta,phi)

subroutine get_energies_angle_pion(n,energy, cos_theta,phi).

The function sigmaMuA_kin() takes as input the atomic number A (integer) of the target nucleus and the incoming muon kinetic energy Ein (double) in $\mathrm{MeV}$ and returns the total muon cross section as calculated using the Weizsäcker-Williams approximation described in Sect. II A. It is a stand-alone function in that it does not require any initializations or previous calls of other functions.

The subroutine scatter muon() takes as input the atomic number A (integer) and proton number Z (integer) of the target nucleus as well as the incoming muon kinetic energy Ein (double) in $\mathrm{MeV}$. It returns particle_flag (integer) and $\mathrm{m}$ (integer). The former can take on only three integer values and is used to tell which type of particle is produced in the reaction: -1 for negative pions, 0 for neutrons, and 1 for positive pions. The returned integer m gives the number of the corresponding particles that is produced. It is also a stand-alone routine, needing no previous initializations or function calls. Note that this routine will only give

one type of emitted particle during a reaction, and not a mixture of particles (e.g. pions and neutrons produced at the same time). This could be an added feature in future modifications of this package.

Given the particle type that is returned in particle_flag, the corresponding call to either get_energies_angles_neutron() or get_energies_angles_pion() should be made. Both these routines need scatter muon() to be called first. These routines take as input the integer $\mathrm{n}$ representing the $\mathrm{n}^{\text {th }}$ emitted particle, where $1 \leq \mathrm{n} \leq \mathrm{m}$. It returns the kinetic energy (double) in $\mathrm{MeV}$, the cosine of the angle relative to the incoming muon direction cos_theta (double), and the azimuthal angle phi. 
When scatter_muon() is called, it first converts the kinetic energy of the incoming muon to a relativistic energy through the relation

$$
E_{i}=m_{\mu} \sqrt{1+2 \operatorname{Ein} / m_{\mu}}
$$

where we have assumed natural units $(c=1)$. It then checks to see if a muon interaction is kinematically allowed. Since we only apply the Weizsäcker-Williams approximation at photon energies above the $\Delta$-resonance $E_{\Delta}$, we must have $E_{i} \geq m_{\mu}+E_{\Delta}$. If this is not satisfied, then the reaction does not occur and Eout is set equal to Ein and $\mathrm{m}$ is set to zero. The routine subsequently exits.

If the reaction is kinematically allowed, the routine then extracts an outgoing muon energy $E_{f}$ by sampling the differential cross section, an example of which was shown in Fig. 3. This sampling procedure is performed by a simple rejection method [14] using a linear distribution as the envelope function. Thus the energy of the exchanged photon $E_{\gamma}$ is determined as $E_{\gamma}=E_{i}-E_{f}$.

To determine whether pions or neutrons are produced during the reaction, the routine calculates $m_{n}$ and $m_{\pi}$ and calculates the ratio $m_{n} /\left(m_{n}+m_{\pi}\right)$. It then generates a random number between 0 and 1 and compares this number to this ratio. If the number is less than the ratio, neutrons are produced, otherwise pions are produced. In the latter case another random number is sampled between 0 and 1 and compared to the ratio $Z / A$. If it is less than this ratio, then $\pi^{-}$particles are produced, otherwise $\pi^{+}$particles are produced. Thus for nuclei in which $Z=A / 2$, on average there will be an equal number of positive and negative pions produced. This is a consequence of assuming isospin invariance.

Since $m_{n}$ and $m_{\pi}$ are in general not whole numbers, the routine chooses $m$ (the number of emitted particles) from nearest integer values that sandwich $m_{n}$ or $m_{\pi}$. It does this at a frequency that would reproduce the average particle multiplicity of $m_{n, \pi}$. As there is no data or theory that gives the width for the distribution of emitted particles about $m_{n, \pi}$, we have adopted this nearest integer procedure. This at least guarantees that we are getting the correct average multiplicity for large samples.

The photon energy $E_{\gamma}$ is split into $E_{\gamma}=E_{k i n}+E_{\text {int }}$, where $E_{k i n}$ represents the amount of energy that is converted into the nucleus' kinetic energy and $E_{\text {int }}$ represents the amount of energy that excites the internal degrees of freedom of the target nucleus. $E_{\text {int }}$ is sampled from a uniform distribution from 0 to $E_{\gamma}[17]$. In the case where pions are emitted, $E_{\text {int }} \geq$ 

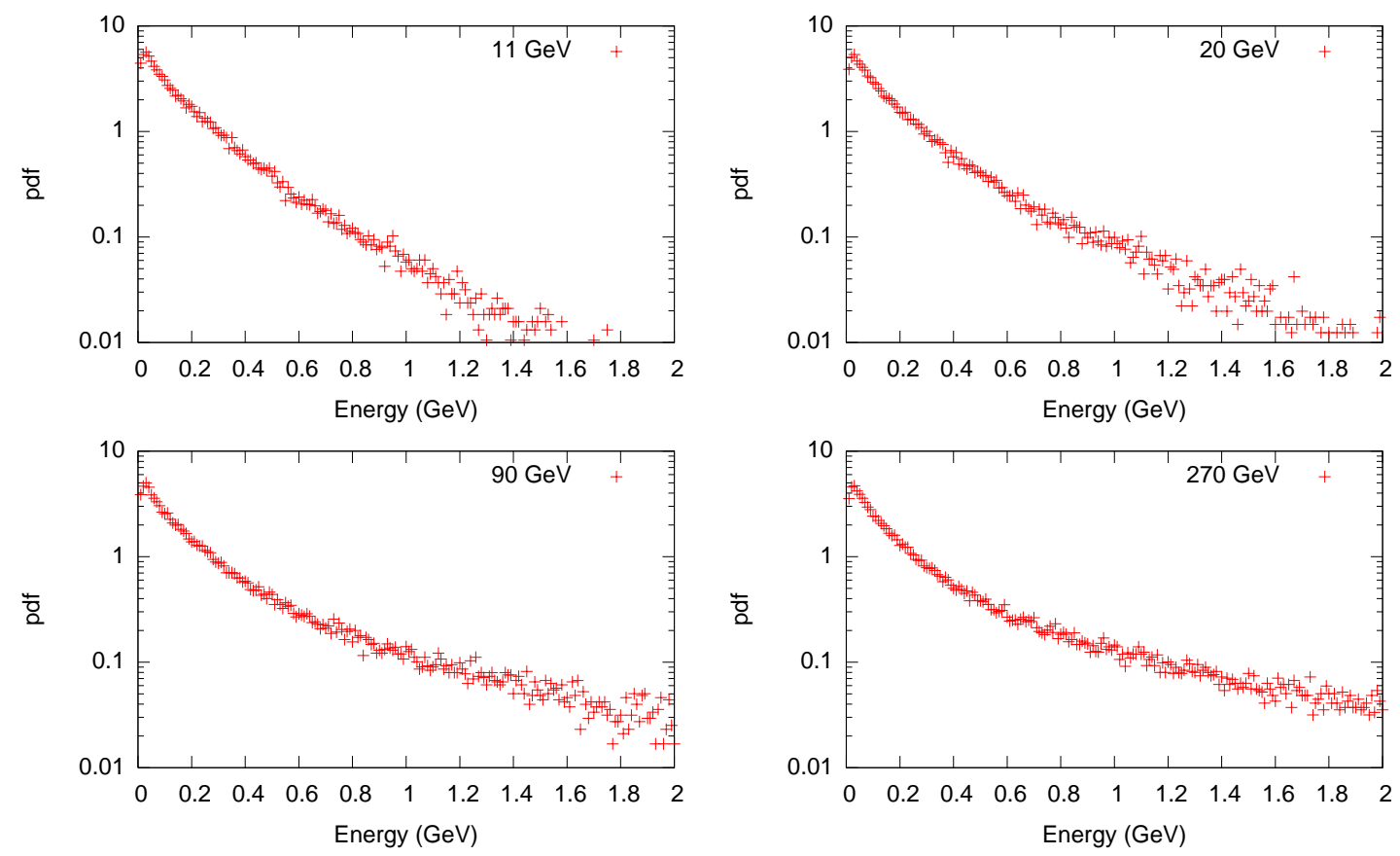

FIG. 6: Primary neutron energy distribution for 11, 20, 90, and $270 \mathrm{GeV}$ incoming muon kinetic energy. The $\mathrm{x}$-axis gives the energy of the outgoing neutrons.

$\mathrm{m} \cdot m_{\mu}$, where $\mathrm{m}$ is the number of emitted pions. This relation is necessary so that energy conservation is preserved. To compute the angles and energies of the outgoing particles we first move to the center of mass (CM) frame where the target nucleus, after interaction with the muon but before emitting any particles, is at rest. Here the initial energy of the nucleus is $M_{A}+E_{\text {int }}$. The directions of the m emitted particles are sampled isotropically, as well as their momenta up to an unknown factor $\alpha$. The direction and momentum of the residual nucleus is chosen so that overall momentum conservation is preserved, up to the unknown factor $\alpha$. Note that the mass of the residual nucleus is $M_{r e s}=M_{A}-\mathrm{m} M_{n}$ for the case of emitted neutrons $\left(M_{n}\right.$ is the nucleon mass), or $M_{r e s}=M_{A}$ for the case of emitted pions. Energy conservation requires that

$$
M_{A}+E_{\text {int }}=\sqrt{M_{r e s}^{2}+\alpha^{2}\left(\sum_{i} p_{i}^{2}+\sum_{i<j} p_{i} p_{j} \cos \left(\theta_{i j}\right)\right)}+\sum_{i} \sqrt{M_{n, \pi}^{2}+\alpha^{2} p_{i}^{2}},
$$

where $\theta_{i j}$ represents the angle between momenta $\mathbf{p}_{\mathbf{i}}$ and $\mathbf{p}_{\mathbf{j}}$ and $M_{n, \pi}$ is either the mass of the pion or neutron, depending on which particle is emitted. A Newton-Raphson iteration method is used to find the value of $\alpha$ which satisfies this equation. Typically only 5-10 

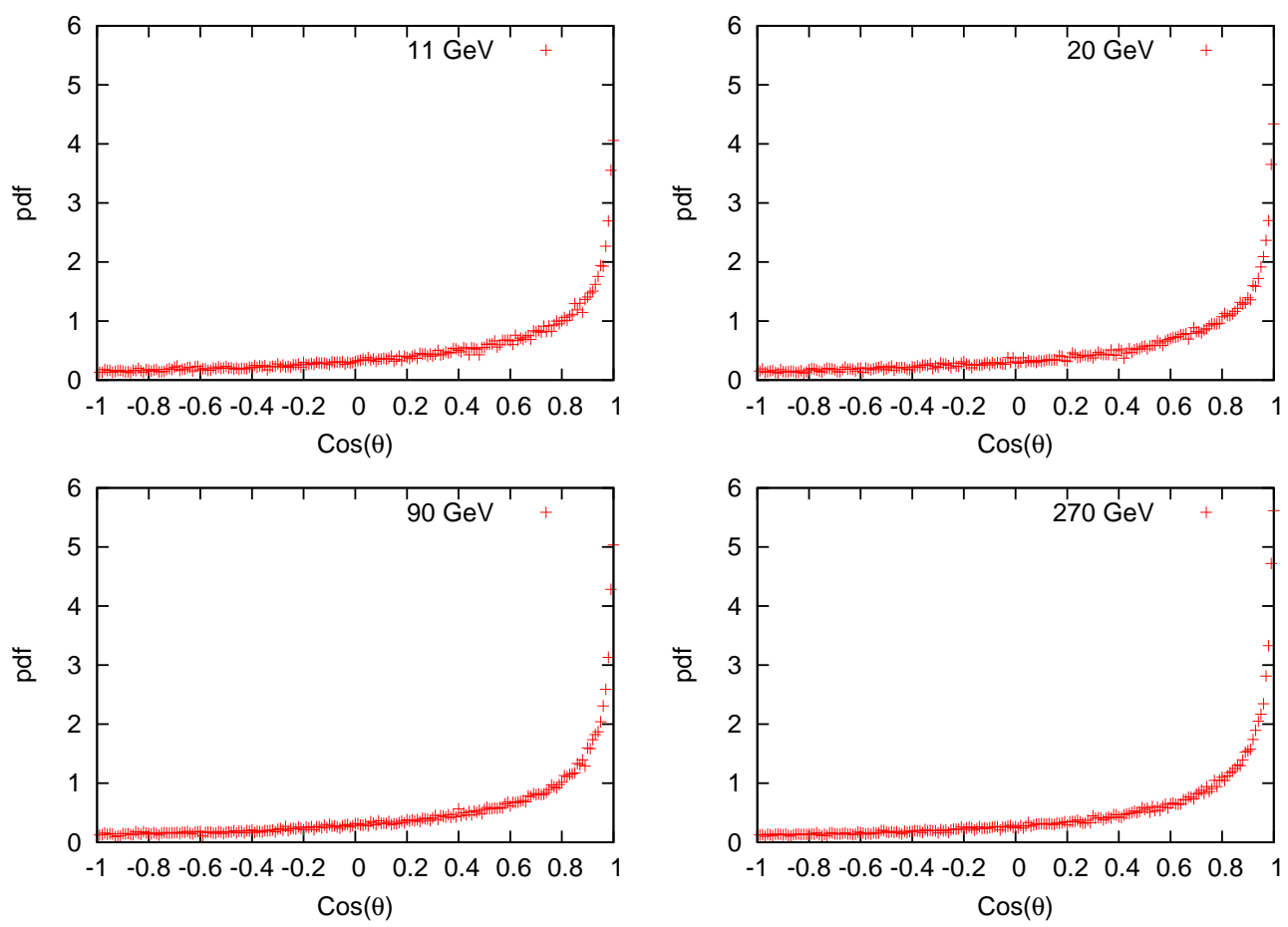

FIG. 7: Primary neutron angular distribution relative to incoming muon direction for 11, 20, 90, and $270 \mathrm{GeV}$ incoming muon kinetic energy.

iterations are needed to get an accuracy to $1 \times 10^{-10}$. Having the momenta and energies of the particles in the CM frame, the system is boosted to the lab frame using the boost factor

$$
\gamma^{2}=1+\left(\frac{E_{k i n}}{M_{A}+E_{i n t}}\right)^{2}+2 \frac{E_{k i n}}{M_{A}+E_{i n t}} .
$$

A linked list is made that stores the energies, angles, and momenta of each of the emitted particles, including those of the residual nucleus. The routines get_energies_angles_pion() and get_energies_angles_neutron() simply access the $\mathrm{n}^{\text {th }}$ element of the linked list to obtain these quantities. With each successive call to scatter_muon(), the linked list is deallocated before being intialized so as to eliminate any possible memory leaks. 

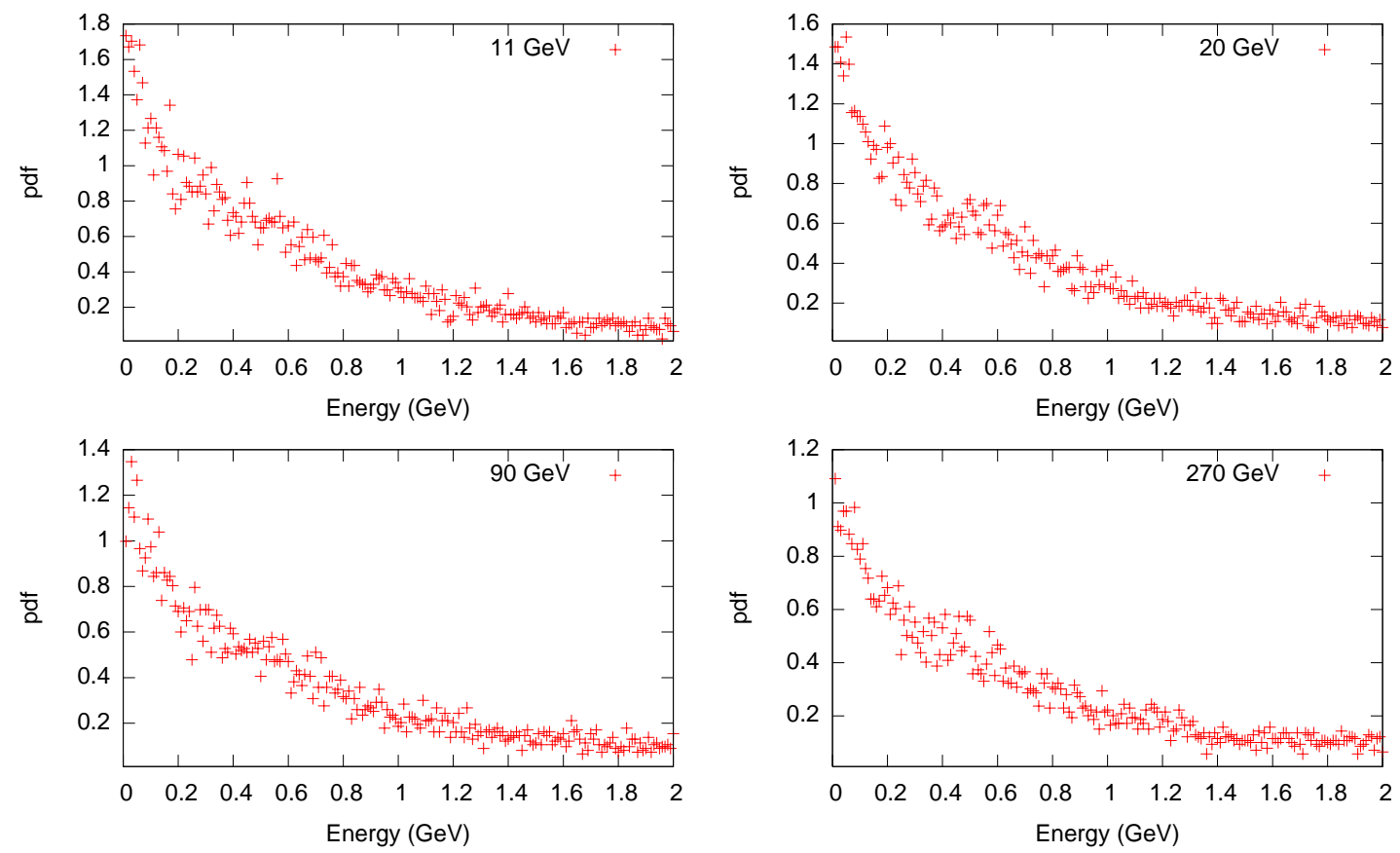

FIG. 8: Primary pion energy distribution for 11, 20, 90, and $270 \mathrm{GeV}$ incoming muon kinetic energy.

\section{RESULTS}

Figure 6 shows the energy distribution (normalized to one) of the primary neutrons produced by muon interactions off Carbon for various incoming muon kinetic energies. Note the decrease in the distribution at small energies, which is not apparent if one includes secondary neutrons (see Fig.4 of Ref. [6]). Figure 7 shows the angular distribution (normalized to one) of the primary neutrons produced from Carbon relative to the direction of the incoming muon for various incoming muon kinetic energies. As noted in Sect. III, the angular distribution in the $\mathrm{CM}$ frame is uniform for $\cos (\theta)$. The skewed distributions shown in Fig. 7 are due to the Lorentz boost to the lab frame.

Figures 8 and 9 show the analogous results for production of primary pions.

\section{CONCLUSION}

Neutron production due to cosmic muon spallation is a constant source of background for long-dwell measurements of fissionable material. As such, it is important to understand 

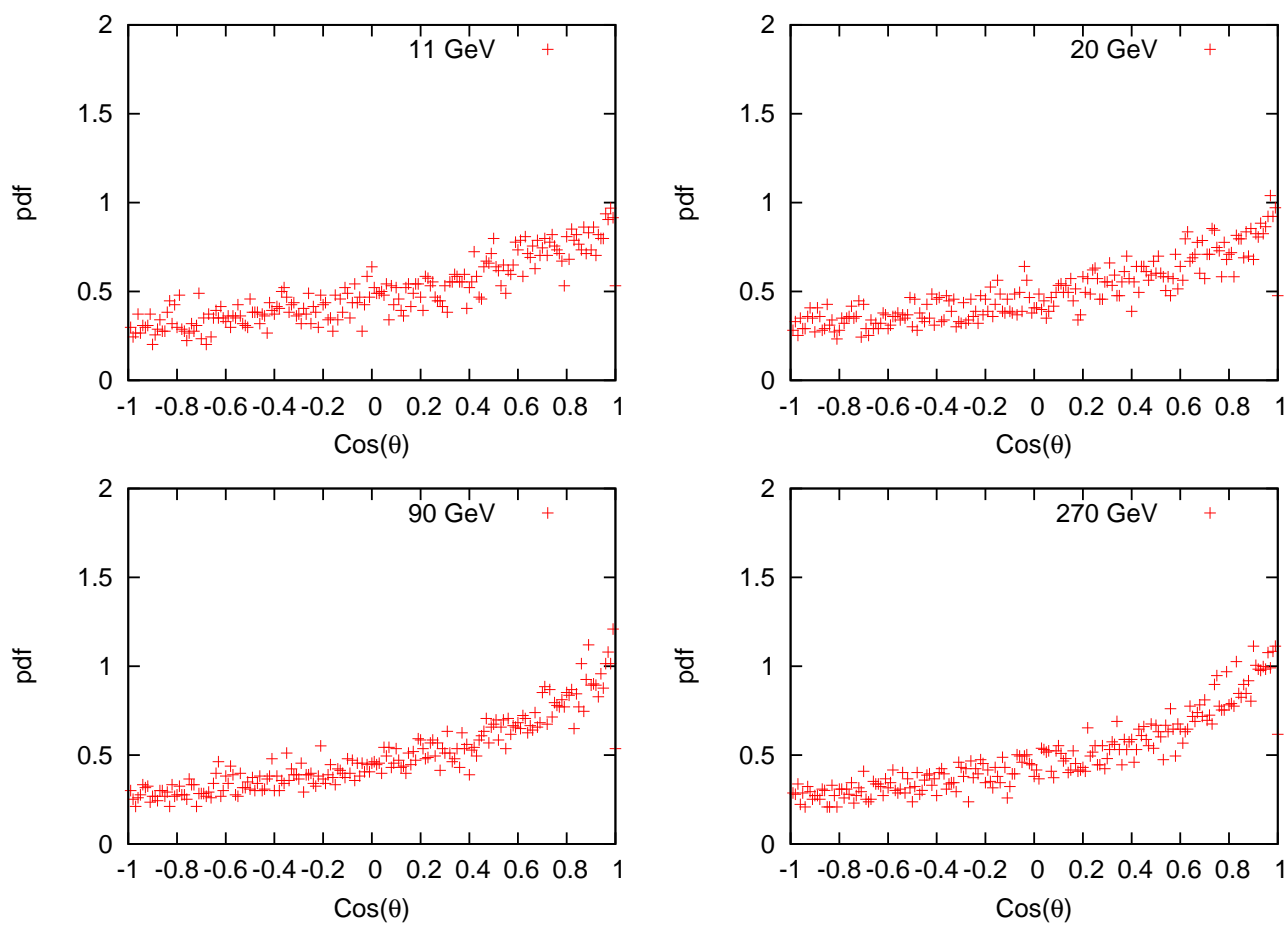

FIG. 9: Primary pion angular distribution relative to incoming muon direction for 11, 20, 90, and $270 \mathrm{GeV}$ incoming muon kinetic energy.

the different underlying physical processes that contribute to neutron production via muon spallation, and their accompanying systematics. Due to the complicated interactions that lead to secondary neutrons, however, a well established theory describing this phenomena is not known. To this end, we have written a muon physics package to be used in conjunction with existing Monte Carlo routines (written in f90). We have calulated neutron and pion multiplicities, as well as energy and angular distributions of primary neutrons and pions. Because the package was developed in-house, the various routines representing the different physical processes can be easily 'switched on' or 'switched off'. This allows for a large degree of control over the code and its various components. This control was lacking in FLUKA. A forthcoming technical report[16] will address the dependence of the calculated spectra on 
secondary processes.

[1] M. Aglietta et al., Nuovo Cimento Soc. Ital. Fis., C 12467 (1989)

[2] F. Boehm et al., Phys. Rev. D 62092005 (2000)

[3] R. Hertenberger et al., Phys. Rev. C 523449 (1995)

[4] O. C. Allkofer and R. D. Andresen, Nucl. Phys B8 402 (1968)

[5] J. Delorme et al., Phys. Rev. C 522222 (1995)

[6] Y.-F. Wang et al., Phys. Rev. D 64013012 (2001)

[7] D. Griffiths, Introduction to Elementary Particles, John Wiley \& Sons, 1987

[8] J. D. Jackson, Classical Electrodynamics, John Wiley \& Sons, 1962

[9] J. D. Walecka, Electron Scattering for Nuclear and Nucleon Structure, Cambridge Monographs 2001

[10] L. B. Bezrukov and E. V. Bugaev, Sov. J. Nucl. Phys. 33635 (1981)

[11] Data on $(\gamma, \mathrm{n})(\gamma, \mathrm{p})$ and $(\gamma, \mathrm{d})$ cross sections, ed. H. Schopper, Springer (1988)

[12] L. Jones and K. Terwilliger, Phys. Rev. 91699 (1953)

[13] P. Kessler, Nuovo Cimento 17809 (1960)

[14] W. Press et al., Numerical Recipes Second Edition, Cambridge Press (1996)

[15] H. Feshback, Theoretical Nuclear Physics: Nuclear Reactions, John Wiley \& Sons (1992)

[16] C. Hagmann and T. Luu, in preparation

[17] In principle, this sampling procedure should be supplanted by a more sophisticated (and hopefully more physical) method, such as one incorporating Hauser-Feshbach theory [15]. 